\title{
Voriconazole-Induced Periostitis Deformans: A Mimicker of Hypertrophic Pulmonary Osteoarthropathy
}

\author{
Karim Ladak, MD and Laurence Rubin, MD
}

\begin{abstract}
$\Lambda$ 30-year-old man with his second double lung transplant for cystic fibrosis presented with 4 months of worsening diffuse bony pain and swelling. He had been receiving oral voriconazole intermittently over 4 years (200mg PO bid) for pulmonary aspergillosis. Examination demonstrated asymmetrical swelling of the phalanges and restricted range of motion in the hips, without clubbing, joint tenderness or tenosynovitis. Hand radiographs showed multifocal, asymmetrical periosteal reaction in the metacarpal shafts and phalanges (Figure 1). MRI demonstrated marked periosteal thickening of the phalangeal diaphyses, with preserved medullary cavities and congruent joints (Figure 2). Bone scan confirmed multifocal osteoblastic activity in the axial and appendicular skeleton (Figure 3). Laboratory findings included an elevated alkaline phosphatase (ALP) [673 IU/L; normal range 35-125] and bone-specific ALP (BS-ALP) [203 mcg/L; normal range 0-20]. Calcium, vitamin $\mathrm{D}$, parathyroid hormone, urate, creatinine, and inflammatory markers were normal. Serum voriconazole and beta-crosslinks (a marker of bone resorption) were normal. Given the elevated ALP, periostitis, osteophytic reactions and lack of clubbing, he was diagnosed with voriconazole-induced periostitis deformans (VIPD). Voriconazole was discontinued and symptoms and ALP improved within 3 months.
\end{abstract}

\section{Discussion}

Voriconazole is a triazole antifungal used to treat invasive fungal infections which are commonly encountered in immunocompromised hosts. Fluoride is a major component of this antifungal, and is central in the pathophysiology of VIPD through stimulation of osteoblasts. ${ }^{1}$ Classically VIPD presents with diffuse bony pain and swelling, elevated serum ALP and fluoride levels, dense periosteal reaction on radiographs, as well as multiple areas of uptake on bone scintigraphy. ${ }^{2,3}$ Despite the aforementioned abnormalities, voriconazole troughs are often within normal limits. ${ }^{4}$ It differs from hypertrophic pulmonary osteoarthropathy in its predilection for distinct areas such as the ribs, irregular and dense periosteal reactions, and lack of clubbing and joint effusions., ${ }^{2,3}$ Symptoms typically resolve soon after discontinuing voriconazole. ${ }^{2,5}$

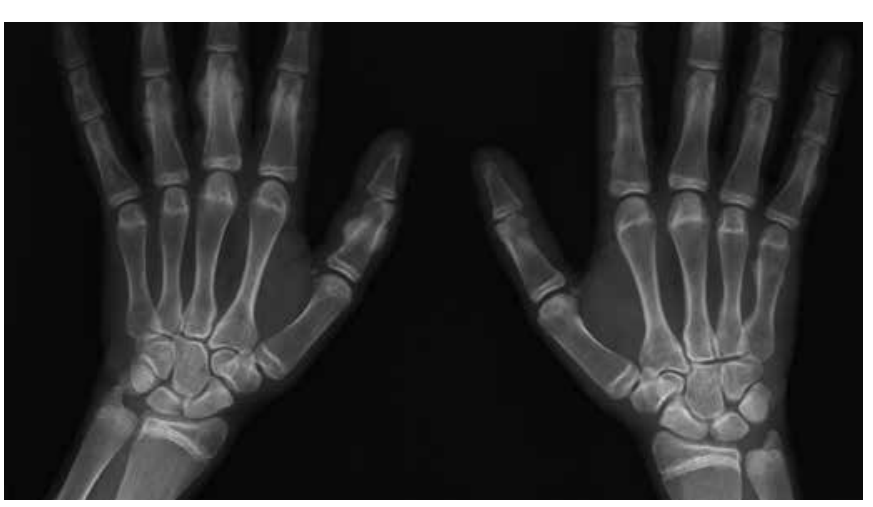

Figure 1. Hand radiographs are notable for multifocal periosteal reaction involving the metacarpals, and proximal and middle phalanges in an asymmetrical manner. The patient is skeletally immature. Anatomic alignment and joint spaces are preserved.

\section{References}

1. Wermers RA, Cooper K, Razonable RR, Deziel PJ, Whitford GM, Kremers WK, Moyer TP. Fluoride excess and periostitis in transplant patients receiving long-term voriconazole therapy. Clin Infect Dis 2011;52:604-611.

2. Wang TF, Wang T, Altman R, Eshaghian P, Lynch JP, Ross DJ, Belperio JA, Weigt SS, Saggar R, Gregson A, Kubak B, Saggar R. Periostitis secondary to prolonged voriconazole therapy in lung transplant recipients. Am J Transplant 2009;9:2845-2850.

3. Chen L, Mulligan ME. Medication-induced periostitis in lung transplant patients: periostitis deformans revisited. Skeletal Radiol 2011;40:143-148.
Corresponding Author: Karim Ladak, MD, Hospital for Special Surgery, Department of Rheumatology, 400 East 7I st street, \#5], New York, NY 1002 I. Email:karladak@gmail.com
Received: February 15, 2017

Accepted: April 24, 2017

doi: $10.3121 / \mathrm{cmr} .2017 .1357$
Keywords: Voriconazole;

Periostitis; Drug Toxicity 


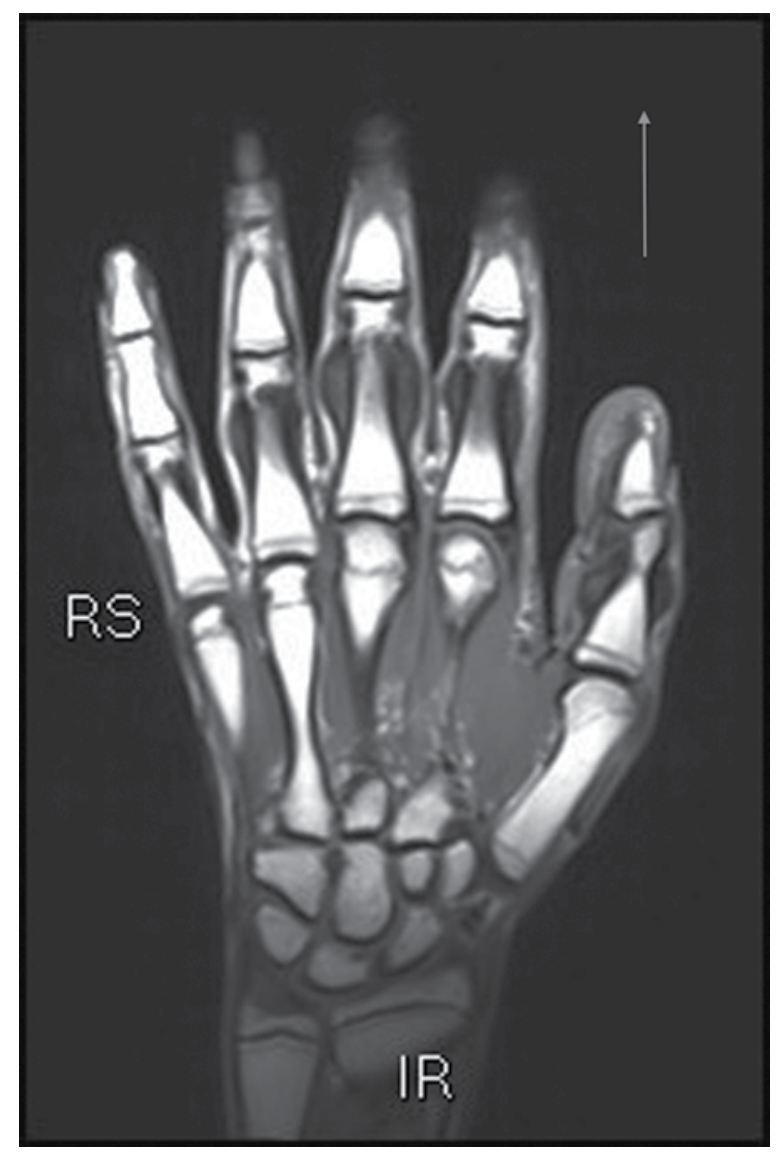

Figure 2. MRI demonstrates marked periosteal thickening involving the diaphyses of the proximal phalanges, as well as more subtle changes at the third middle phalanx. This is T2 hyperintense and T1 hypointense to background marrow, suggesting immature callus formation. The underlying medullary cavity is preserved.

4. Moon WJ, Scheller EL, Suneja A, Livermore JA, Malani AN, Moudgal V, Kerr LE, Ferguson E, Vandenberg DM. Plasma fluoride level as a predictor of voriconazole-induced periostitis in patients with skeletal pain. Clinical Infectious Disease 2014; 59(9);1237-1245.

5. Gerber B, Guggenberger R, Fasler D, Nair G, Manz MG, Stussi G, Schanz U. Reversible skeletal disease and high fluoride serum levels in hematologic patients receiving voriconazole. Blood 2012;120:2390-2394.

\section{Author Affiliations}

Karim Ladak, MD* and Laurence Rubin, MD†

*Clinical Fellow, Hospital for Special Surgery, Rheumatology Department, New York, New York, USA †Professor of Medicine, University of Toronto, Rheumatology Department, Toronto, Canada
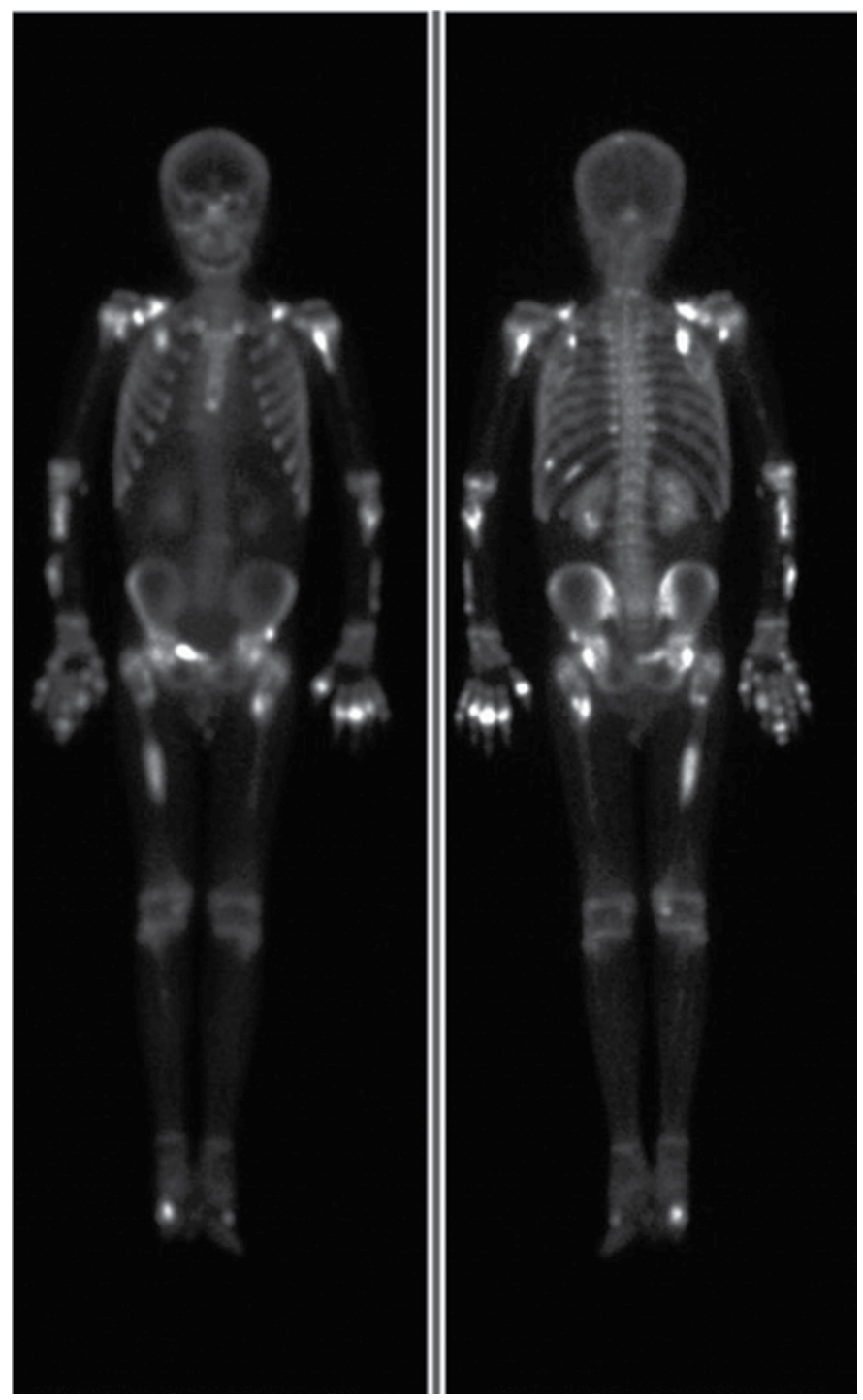

Figure 3. Delayed total body skeletal phase images on bone scan demonstrate multiple sites of increased activity throughout the skeleton. These sites include the midfeet, femurs, right pubic bone, the left superior acetabular lip, radius, ulna and humeral heads bilaterally. The hands and fingers, several ribs, clavicles and small sites in the skull were also affected. The spine and sternum were relatively spared. 